\title{
Photosynthesis gets a boost
}

The summer sun is shining bright when a small cloud dims its glow. Below in the field, acres of planted crops adjust to the sudden change in light intensity-a slow transition that will cost the plants dearly in photosynthetic efficiency. But recent work by Kromdijk et al. ${ }^{1}$ argues plants can be engineered to adjust to fluctuating light at a faster rate. Writing in Science, the researchers show that modifications in this pathway can result in a remarkable increase in plant dry weight yield-about $15 \%$-both in the laboratory and in the field.

When a plant is exposed to more light than it can use in photosynthesis, the excess energy is dissipated in the form of heat. This mechanism of photoprotection is critical for avoiding photodamage that can be caused by the accumulation of harmful reactive oxygen species. But when light intensity is reduced (by cloud cover or wind-driven changes in shadowing by other leaves, for example), dissipation of radiant energy is no longer necessary. Unfortunately, although photoprotection mechanisms kick in quickly, they can take minutes or even hours to revert back once they are no longer needed. The continued dissipation of light energy while the plant is in the shade is estimated to reduce $\mathrm{CO}_{2}$ fixation by up to $30 \%$.

Aiming to modulate the kinetics of the xanthophyll cycle, which influences energy dissipation, Kromdijk et al. ${ }^{1}$ introduced genes encoding additional copies of three key cycle enzymes into tobacco plants, effectively increasing their level of expression. This approach sped up the rate of induction and relaxation of the photoprotection cycle. Under constant light, both engineered and wild-type plants behaved similarly, but in fluctuating light, photosynthetic

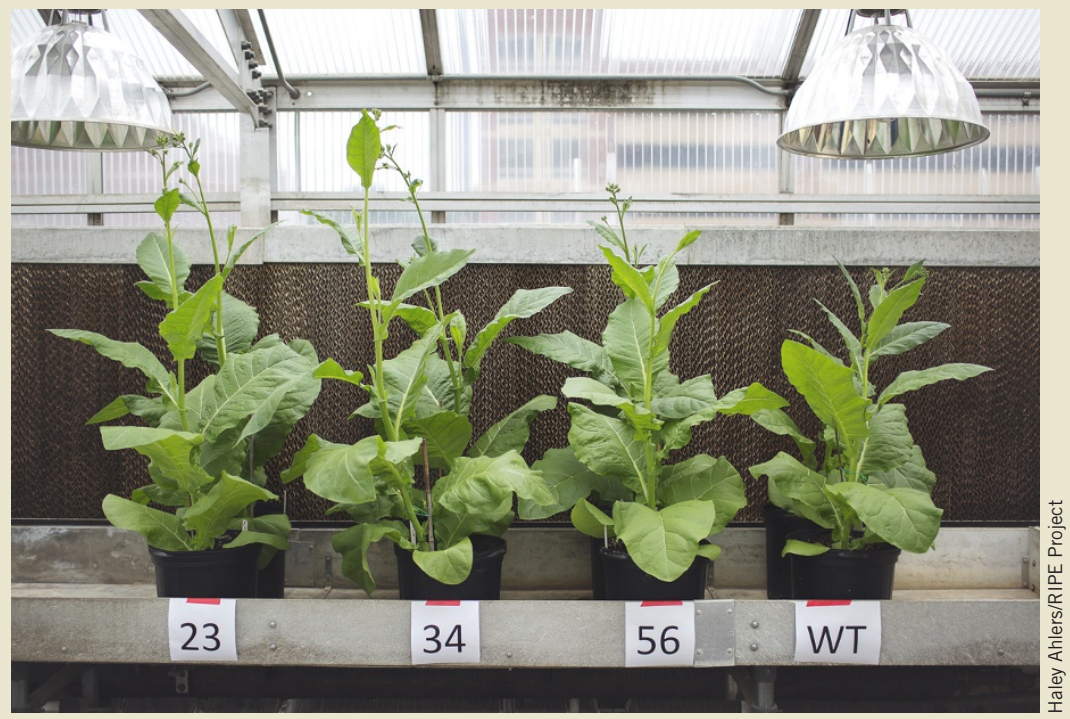

$\mathrm{CO}_{2}$ assimilation increased by up to $14 \%$ in modified plants. This translated to a substantial effect in yield: $14-20 \%$ greater total dry weight per plant in field trials.

"You would think maybe what you should do is dial down the photoprotection mechanism, but that can have obvious negative effects because you need that photoprotection when you are in full sun," says Robert Blankenship, a Distinguished Professor of Arts and Sciences at Washington University in St. Louis. "By amplifying the whole pathway and not just one part of it, the relative functionality of the pathway remained intact, but the response time to changes in light conditions was decreased," he explains. Importantly, the researchers didn't observe increased photodamage after exposing the modified plants to excessive light for 2 hours.

Because the xanthophyll cycle is remarkably well-conserved among higher plants, it is likely that the findings will translate to plants besides the tobacco model, potentially including crops such as soy, wheat and rice. "The results are exciting," says Blankenship, "because they indicate that there is potential for significant improvement of photosynthesis efficiency and crop productivity that is not yet realized." There are other avenues to improve photosynthetic yield that are actively being studied but have not proven successful yet. Achieving a more even distribution of light across all leaves in the plant by antenna reduction, expanding the light spectrum that a plant can absorb into the near-infrared range and engineering the carbon reduction pathway are just some of the possibilities, says Blankenship.

Irene Jarchum, Associate Editor 1. Kromdijk, J. et al. Science 354, 857-860 (2016).

\section{Research Highlights}

Papers from the literature selected by the Nature Biotechnology editors. (Follow us on Twitter, @NatureBiotech \#nbtHighlight)

Discovery of MRSA active antibiotics using primary sequence from the human microbiome Chu, J. et al. Nat. Chem. Biol. 12, 1004-1006 (2016)

Cell-type-specific optical recording of membrane voltage dynamics in freely moving mice Marshall, J.D. et al. Cell 167, 1650-1662.e15 (2016)

Generation of influenza A viruses as live but replication-incompetent virus vaccines

Si, L. et al. Science 354, 1170-1173 (2016)

Genetic drivers of epigenetic and transcriptional variation in human immune cells Chen, L. et al. Cell 167, 1398-1414.e24 (2016)

Phantom motor execution facilitated by machine learning and augmented reality as treatment for phantom limb pain: a single group, clinical trial in patients with chronic intractable phantom limb pain

Ortiz-Catalan, M. et al. Lancet doi:10.1016/S0140-6736(16)31598-7 (2016) 